\title{
Mycobacterial escape artists
}

DOI:

10.1038/nrmicro1728

\section{URLs}

Mycobacterium tuberculosis http://www.ncbi.nlm.nih.gov/ sites/entrez?db=genomeprj\& $\mathrm{md}=$ Retrieve\&dopt=Overview Elist_uids $=12318$

Mycobacterium leprae http://www.ncbi.nlm.nih.gov/ sites/entrez?Db=genomeprj\& $\mathrm{md}=$ ShowDetailView\&TermTo Search $=90$

BCG

http://www.ncbi.nlm.nih.gov/ sites/entrez?db=genomeprj\&c $\mathrm{md}=$ Retrieve\&dopt=Overview Elist_uids= 12311
Reporting in Cell, van der Wel et al. have provided evidence that Mycobacterium tuberculosis and Mycobacterium leprae do not replicate in intracellular phagosomes, as had previously been thought, but instead replicate in the cytosol of myeloid cells after they translocate from phagolysosomes.

Microorganisms that are engulfed by myeloid cells such as macrophages are internalized into membrane-bound organelles called phagosomes. The microorganisms are then destroyed in the acidic environment that is created when the phagosomes are delivered to and fuse with lysosomes. Intracellular pathogens avoid lysosomal fusion either by manipulating the host and creating privileged endosomal replicative niches, or by escaping from the endocytic system and replicating in the cytosol. Although there has been some evidence that free $M$. tuberculosis is present in the cytoplasm, both M. tuberculosis and M. leprae were thought to be prototypical 'endosomal' pathogens, until now.

Most experimental systems that are used to study the localization of mycobacteria in infected macrophages have focused on the first 48 hours after infection. Here, the authors extended this investigation time to 7 days and used a technique called cryo-immunogold electron microscopy. They initially found that M. tuberculosis and M. leprae localize to a phagolysosomal compartment in dendritic cells (DCs) and macrophages soon after phagocytosis. Further investigation with $M$. tuberculosis indicated that the mycobacteria are probably not exposed to degradation in $76 \%$ of phagolysomes that contain M. tuberculosis. So how do the mycobacteria avoid acidification and degradation in these compartments? Although M. tuberculosis persisted during the initial 48-hour infection period in DCs, they were only able to replicate significantly after this time point. The authors found that this was because a large subset of intracellular $M$. tuberculosis resides in the cytosol of a large proportion of DCs at later timepoints after infection. The fact that the translocation of mycobacteria into the host cytosol occurred in non-apoptic cells eliminated the possibility that the bacteria appeared in the cytosol because of the reduced viability of infected DCs.

Entry into the cytosol of infected cells was also shown for M. leprae, but not for heatkilled mycobacteria (indicating that cytosolic entry requires an active mycobacterial process) or for the non-pathogenic strain, Mycobacterium bovis bacillus Calmette-Guérin (BCG), which is used in the vaccine for tuberculosis (indicating that translocation to the cytosol is a feature of virulent mycobacteria). A requirement was also shown for the immunogenic bacterial proteins early secreted antigenic target 6 (ESAT6) and culture filtrate protein 10 (CFP10), which are absent from M. bovis BCG. Finally, the authors showed that the translocation of M. tuberculosis to the cytosol is followed by apoptosis of the infected cell.

So, van der Wel et al. have shown that M. tuberculosis and M. leprae egress from the endocytic system and replicate in the cytosol. As $M$. bovis BCG does not translocate, this could explain why the BCG vaccine is not effective against adult pulmonary tuberculosis - it may be that translocation to the cytosol is required to stimulate crucial $\mathrm{CD}^{+}$ T-cell responses through major histocompatibility complex class I. This work, therefore, has implications for the design of improved vaccines for pulmonary tuberculosis.

Sharon Ahmad

ORIGINAL RESEARCH PAPER van der Wel, N. et al. M. tuberculosis and M. leprae translocate from the phagolysosome to the cytosol in myeloid cells. Cell 129, 1287-1298 (2007)

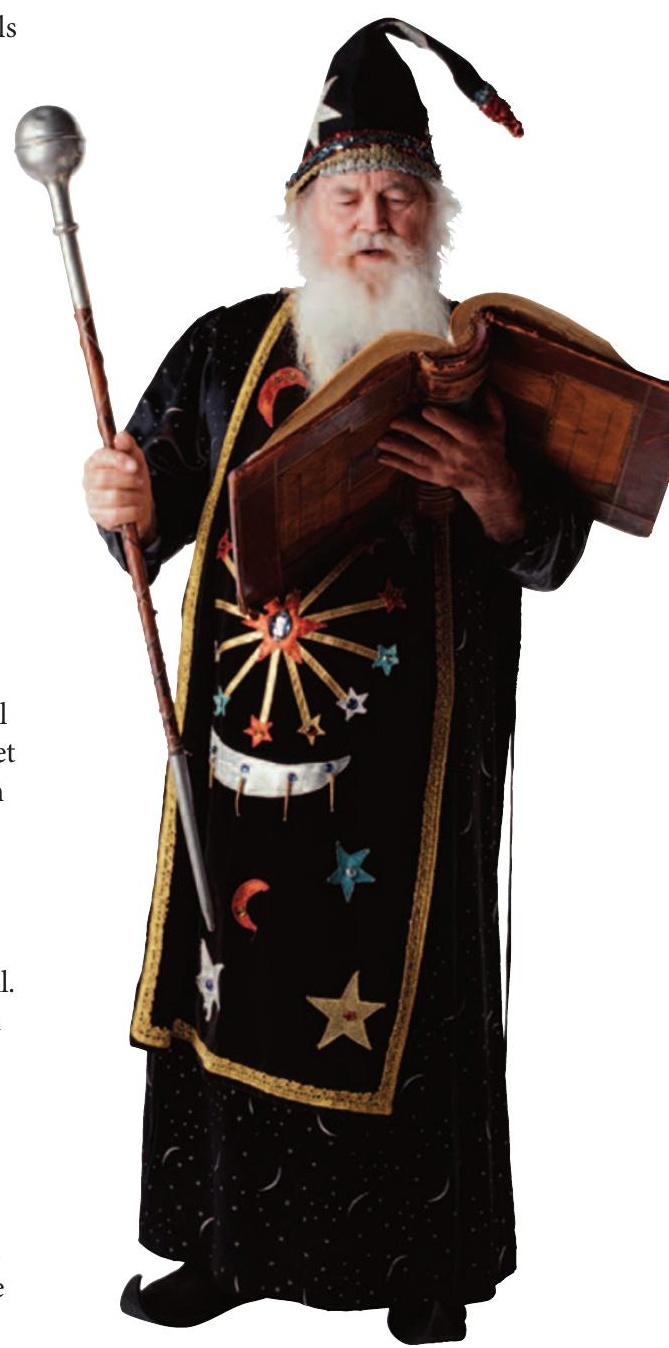

